\title{
Quercetin Induces Mitochondrial Biogenesis through Activation of HO-1 in HepG2 Cells
}

\author{
Nabin Rayamajhi, ${ }^{1,2}$ Seul-Ki Kim, ${ }^{1}$ Hiroe Go, ${ }^{3}$ Yeonsoo Joe, ${ }^{1}$ Zak Callaway, ${ }^{1}$ Jae-Gu Kang, \\ Stefan W. Ryter, ${ }^{5}$ and Hun Taeg Chung ${ }^{1}$ \\ ${ }^{1}$ School of Biological Sciences, University of Ulsan, Ulsan 680-749, Republic of Korea \\ ${ }^{2}$ Chitwan Medical College, Bharatpur-10, Chitwan, Nepal \\ ${ }^{3}$ Korea Bio Medical Science Institute, Seoul 135-818, Republic of Korea \\ ${ }^{4}$ Graduate School of Oriental Medicine, University of Wonkwang, Iksan 570-300, Republic of Korea \\ ${ }^{5}$ Department of Pulmonary and Critical Care Medicine, Brigham and Women's Hospital, Harvard Medical School, \\ Boston, MA 02115, USA
}

Correspondence should be addressed to Hun Taeg Chung; chung@ulsan.ac.kr

Received 19 July 2013; Accepted 23 September 2013

Academic Editor: Hong Pyo Kim

Copyright (C) 2013 Nabin Rayamajhi et al. This is an open access article distributed under the Creative Commons Attribution License, which permits unrestricted use, distribution, and reproduction in any medium, provided the original work is properly cited.

\begin{abstract}
The regeneration of mitochondria by regulated biogenesis plays an important homeostatic role in cells and tissues and furthermore may provide an adaptive mechanism in certain diseases such as sepsis. The heme oxygenase (HO-1)/carbon monoxide (CO) system is an inducible cytoprotective mechanism in mammalian cells. Natural antioxidants can provide therapeutic benefit, in part, by inducing the HO-1/CO system. This study focused on the mechanism by which the natural antioxidant quercetin can induce mitochondrial biogenesis in HepG2 cells. We found that quercetin treatment induced expression of mitochondrial biogenesis activators (PGC-1 $\alpha$, NRF-1, TFAM), mitochondrial DNA (mtDNA), and proteins (COX IV) in HepG2 cells. The HO inhibitor SnPP and the CO scavenger hemoglobin reversed the effects of quercetin on mitochondrial biogenesis in HepG2 cells. The stimulatory effects of quercetin on mitochondrial biogenesis could be recapitulated in vivo in liver tissue and antagonized by SnPP. Finally, quercetin conferred an anti-inflammatory effect in the liver of mice treated with LPS and prevented impairment of mitochondrial biogenesis by LPS in vivo. These salutary effects of quercetin in vivo were also antagonized by SnPP. Thus, our results suggest that quercetin enhances mitochondrial biogenesis mainly via the HO-1/CO system in vitro and in vivo. The beneficial effects of quercetin may provide a therapeutic basis in inflammatory diseases and sepsis.
\end{abstract}

\section{Introduction}

Mitochondrial biogenesis plays an important role in cell survival and repair [1-3]. Increased oxidative damage and inflammation can cause mitochondrial damage that may lead to serious acute and chronic pathologies such as multiorgan failure, neurodegeneration, and cardiovascular disease [36]. Mitochondrial biogenesis can enhance cellular function and survival in vivo and in vitro and promote cellular recovery from damage caused by adverse environmental, pathophysiological, and/or infectious agents $[7,8]$.

Mitochondrial biogenesis is regulated by a complex network of factors. The peroxisome proliferator-activated receptor gamma coactivator (PGC) family of transcription co-activators (e.g., PGC- $1 \alpha$ ) coactivate nuclear respiratory factor 2 (NRF-2/GA-Binding protein-A) and nuclear respiratory factor-1 (NRF-1) $[1,9]$. PGC- $1 \alpha$ and NRF- 1 activate mitochondrial transcription factor A (TFAM) that is responsible for transcribing nuclear encoded mitochondrial proteins, including structural proteins as well as proteins involved in mitochondrial DNA (mtDNA) transcription, translation, and repair [1, 2, 8-11].

Quercetin is a naturally occurring flavonoid which has a broad spectrum of bioactive effects. Among these, quercetin can impact mitochondrial biogenesis by modulating enzymes and transcription factors in the inflammatory signaling 
cascade $[10,12]$. Previous studies have shown that quercetin can increase messenger RNA (mRNA) for PGC- $1 \alpha$, the cytosolic deacetylase SirtI, and cytochrome $c$ concentration in soleus muscles [13]. Quercetin, a potent phenolic antioxidant, can also modulate mitochondrial biogenesis by reducing ROS production in various cell types $[14,15]$. Mitochondrial ROS can perturb cellular oxidant/antioxidant balance and participate in redox signaling. Oxidative stress-related ROS production can stimulate adaptive responses, such as Nrf2 translocation and binding to antioxidant response element (AREs) motifs in protective phase II antioxidant genes including heme oxygenase-1 (Hmox1). However, increased ROS production can cause mitochondrial dysfunction and cell death $[9,16]$. Polyphenol antioxidants can prevent ROSinduced cellular damage by scavenging free radicals. The process of excess ROS elimination and mitochondrial biogenesis is connected with innate cellular antioxidant defense mechanisms.

Heme oxygenase-1 (HO-1) is an important antioxidant enzyme that catalyses the rate-limiting step in hemedegradation. HO-1 induction protects against prooxidant heme release induced by many agents like LPS, cytokines, and ROS. Degradation of heme results in production of biliverdin, iron, and $\mathrm{CO}$ which have important physiological effects. Biliverdin is converted to the potent endogenous antioxidant bilirubin by NADPH: biliverdin reductase. Humans with HO-1 deficiency exhibit severe medical conditions such as anemia, leukocytosis, and hyperlipidemia, while animal models with HO-1 deficiency are susceptible to endotoxemia and chronic hypoxia $[17,18]$. HO-1 deficient endothelial cells display increased injury in the presence of oxidative challenge, suggesting that the $\mathrm{HO}-1$ pathway is a key cytoprotective mechanism against oxidative stress which contributes to cellular homeostasis [11, 17-19].

Endogenous CO contributes to the protective effects of HO-1 by modulation of the inflammatory response. CO binds to cytochrome $c$ oxidase resulting in increased mitochondrial ROS production, which enhances mitochondrial biogenesis. Limited bioavailability of CO by hemoglobin treatment triggers cell death with a concomitant decline in ATP production, and mitochondrial generation of ATP significantly declined when $\mathrm{CO}$ availability was limited. These results suggest that $\mathrm{CO}$, an enzymatic byproduct of $\mathrm{HO}-1$ activity, is responsible for the function of $\mathrm{HO}-1$ and that the $\mathrm{HO}-1 / \mathrm{CO}$ system may preserve mitochondrial biogenesis [17-20].

In the current study we demonstrate the role of the $\mathrm{HO}-1 / \mathrm{CO}$ system in mediating mitochondrial biogenesis induced by the antioxidant quercetin in HepG2 cells. An understanding of the mechanisms underlying mitochondrial biogenesis may facilitate the development of therapeutics in diseases involving mitochondrial dysfunction (e.g., sepsis, and metabolic syndrome).

\section{Materials and Methods}

2.1. Reagents. Quercetin, Hemoglobin ( $\mathrm{Hb})$, and bacterial lipopolysaccharide (LPS, from Escherichia coli 055:B5) were purchased from Sigma-Aldrich (St Louis, MO). Tin
protoporphyrin-IX (SnPP) was from Porphyrin Products Inc. (Logan, UT). Antibodies against $\beta$-actin were purchased from Santa Cruz Biotechnology (Santa Cruz, CA), and antibodies to cytochrome oxidase subunit IV (COX) IV and $\alpha$ tubulin were purchased from Cell Signaling (Danvers, MA). Antibody against HO-1 was purchased from Assay Designs (Ann Arbor, MI). All other chemicals were purchased from Sigma-Aldrich.

2.2. Cell Culture and Quercetin Treatment. HepG2 cells were purchased from ATCC (Manassas, VA). Cells were cultured in DMEM media supplemented with $10 \%$ fetal bovine serum, $100 \mathrm{U} / \mathrm{mL}$ penicillin, and $100 \mathrm{mg} / \mathrm{mL}$ streptomycin (Gibco, $\mathrm{NY}$ ). Cells were maintained in a humidified incubator at $37^{\circ} \mathrm{C}$ under an atmosphere of $5 \% \mathrm{CO}_{2}$. For quercetin treatment, HepG2 cells $\left(4 \times 10^{4}\right.$ cells/well $)$ were grown on 6 -well plates overnight and quercetin was administered at various doses (5-25 $\mu \mathrm{M})$ and times (3-24 hrs).

2.3. Animals. All experiments with mice were approved by the Animal Care Committee of the University of Ulsan. Seven week-old male C57BL/6 mice were purchased from ORIENT (Pusan, Korea). The mice were maintained under specific pathogen-free conditions at $18-24^{\circ} \mathrm{C}$ and $40-70 \%$ humidity, with a $12 \mathrm{~h}$ light-dark cycle, and food and drinking water were available ad libitum.

C57BL/6 mice were treated with an intraperitoneal (i.p.) injection of quercetin $(50 \mathrm{mg} / \mathrm{kg})$ dissolved in $0.5 \%$ DMSO/PBS solution for seven alternate days. The control group of mice received the same amount of $0.5 \% \mathrm{DMSO} / \mathrm{PBS}$ solution. In some experiments, SnPP $(50 \mu \mathrm{mol} / \mathrm{kg})$ was administered intraperitoneally (i.p.) to mice before quercetin injection. SnPP was dissolved in $0.1 \mathrm{~N} \mathrm{NaOH}$ and diluted with PBS ( $\mathrm{pH}$ 7.4). To study sepsis in mice, twenty-four hours after the final injection of quercetin, mice received an injection of LPS (10 mg/kg, i.p.). At $24 \mathrm{~h}$ after LPS injection, mice were sacrificed under anesthesia and liver tissue was harvested for RNA, mtDNA, and protein measurements.

2.4. Reverse Transcription Polymerase Chain Reaction (RT$P C R)$. Total RNA of HepG2 was extracted using Trizol reagent (Invitrogen, CA). Two micrograms of total RNA were used for reverse transcription polymerase chain reaction (RTPCR) analysis using oligo-dT primers (Qiagen, CA) and MMLV reverse transcriptase (Promega, WI) according to the manufacturer's instructions. The forward and reverse primers used in the present study are shown in Table 1. PCR products were electrophoresed on 1.5\% agarose gel and visualized by ethidium bromide staining. GAPDH cDNA level was used as an internal control.

2.5. Western Blotting Analysis. Cells were harvested in lysis buffer [25 mMTris- $\mathrm{HCl}$ (pH 7.5), $137 \mathrm{mM} \mathrm{NaCl,} 2.7 \mathrm{mM}$ $\mathrm{KCl}, 1 \%$ Triton $\mathrm{X}-100$ ] containing protease and phosphatase inhibitors cocktail (Sigma-Aldrich, St. Louis, MO). Protein concentration was measured with BCA protein assay reagent (Pierce, Rockford, IL). Equal amounts of proteins were separated using SDS-PAGE and transferred to polyvinylidene 
TABLE 1: Gene primers used in this study.

\begin{tabular}{lrr}
\hline Gene & Forward primer $\left(5^{\prime}-3^{\prime}\right)$ & Reverse primer $\left(5^{\prime}-3^{\prime}\right)$ \\
\hline hPGC1 $\alpha$ & GGAACTGCAGGCCTAACTCC & CACTGTCCCTCAGTTCACCG \\
hNRF- 1 & CCAGTGGCCACACAGAACTC & CTTCCTTTCCCTTCCACTGC \\
hTfam & ATGCTTATAGGGCGGAGTGG & TGGTTTCCTGTGCCTATCCA \\
hHO-1 & GGAACTTTCAGAAGGGCCAG & GTCCTTGGTGTCATGGGTCA \\
hGAPDH & GGGGCTCTCCAGAACATCAT & TCAAGGGGTCTACATGGCAA \\
mPGC-1 $\alpha$ & GGAACTGCAGGCCTAACTCC & TTGGAGCTGTTTTCTGGTGC \\
mNRF-1 & CTCCAAACCCAACCCTGTCT & TGGTGGCCTGAGTTTGTGTT \\
mTfam & CAGCCAGGTCCAGCTCACTA & ATTAGGAGGGTCTCGCTCCA \\
mTNF- $\alpha$ & AGACCCTCACACTCAGATCATCTTC & TTGCTACGACGTGGGCTACA \\
mIL-1 $\beta$ & TCGCTCAGGGTCACAAGAAA & ATCAGAGGCAAGGAGGAAACAC \\
m $18 \mathrm{~s}$ & CCAGAGATACAAAGAAATGATGG & ACTCCAGAAGACCAGAGGAAAT \\
\hline
\end{tabular}

difluoride membranes (Thermo Scientific, Rockford, IL). Membranes were blocked with 5\% skim milk in PBS containing $0.1 \%$ Tween 20 (PBS-T) for $1 \mathrm{~h}$ and then incubated with the specified antibodies. Signals were detected using the ECL detection system (Thermo Scientific, Rockford, IL, USA).

2.6. Quantitative Real-Time PCR Analysis of mt DNA Content. Genomic DNA (containing both mitochondrial and nuclear DNA) was isolated from cells using a Blood and Cell Culture DNA Mini Kit (Qiagen, Valencia, CA) according to the manufacturer's instructions. mtDNA was determined by SYBR green quantitative PCR (qPCR). The following primers for mtDNA were used: Human Complex II (succinateubiquinone oxidoreductase): forward primer $5^{\prime}$-CAAACCTACGCCAAAATCCA-3' reverse primer $5^{\prime}$-GAAATGAATGAGCCTACAGA-3'. Mouse cytochrome $b$ (Mus musculus domesticus mitochondrion): forward primer $5^{\prime}$-CCACTTCATCTTACCATTTA- $3^{\prime}$ reverse primer $5^{\prime}$-ATCTGCATCTGAGTTTAATC- $3^{\prime}$. The following primers for nuclear DNA were used: human $\beta$-actin: forward primer $5^{\prime}$-TCACCCACACTGTGCCCATCTACGA-3 ${ }^{\prime}$ reverse primer $5^{\prime}$ CAGCGGAACCGCTCATTGCCAATGG- $3^{\prime}$ and mouse $18 \mathrm{~S}$ rRNA: forward primer $5^{\prime}$-GGGAGCCTGAGAAACGGC$3^{\prime}$ reverse primer $5^{\prime}$-GGGTCGGGAGTGGGTAATTT-3'. Reactions were performed with SYBR Green qPCR Master Mix (2X; USB production, Affymetrix) on an ABI 7500 Fast Real-Time PCR System (Applied Biosystems, Carlsbad, CA).

2.7. Statistical Analysis. Multiple mean values were compared using analysis of variance (ANOVA) with GraphPad Prism. Values presented are mean \pm SD. ANOVA using $t$-tests was applied to compare the mean of each group with that of the control group. A $P<0.05$ was considered to be statistically significant.

\section{Results}

3.1. Quercetin Induces the Expression of Activators and Mitochondrial Proteins Associated with Mitochondrial Biogenesis.
We determined the potential of quercetin to induce mitochondrial biogenesis by analyzing the mRNA expression levels of major regulators of mitochondrial biogenesis (i.e., PCG- $1 \alpha$, NRF-1, and TFAM). Treatment of HepG2 cells with quercetin $(15 \mu \mathrm{M})$ significantly increased the levels of PGC$1 \alpha$, NRF-1, and TFAM mRNA in a time-dependent and dose-dependent manner (Figures 1(a) and 1(b)). Quercetin treatment also stimulated the expression of the major mitochondrial protein COX IV in a time- and dose-dependent manner (Figures 1(c) and 1(d)). Since increases of mtDNA copy number also represent an index of mitochondrial biogenesis, we measured the amount of mtDNA in HepG2 cells treated with quercetin (Figures 1(e) and 1(f)). Quercetin increased mtDNA copy number with an apparent maximum at a quercetin dose of $15 \mu \mathrm{M}$ for 3 hrs.

3.2. Quercetin Induces Mitochondrial Biogenesis via Expression of the HO-1/CO System in HepG2 Cells. Quercetin has previously been shown to induce $\mathrm{HO}-1$ expression in various cell types, which may account in part for the cytoprotective, antiapoptotic, antioxidant, and anti-inflammatory effects of this compound $[19,21-23]$. In the current study, we examined whether quercetin can induce the expression of HO1 at the RNA or protein level in HepG2 cells. As shown in Figure 2(a), an increase in $\mathrm{HO}-1 \mathrm{mRNA}$ and protein was detected at various times and doses of quercetin. The maximal effect of quercetin on HO-1 mRNA and protein expression was observed after treatment with $15 \mu \mathrm{M}$ for $3 \mathrm{~h}$ (Figure 2(a), left and middle). When HepG2 cells were treated with different concentrations of quercetin $(5-25 \mu \mathrm{M})$ for $3 \mathrm{hrs}$, the maximum induction of HO-1 protein was detected at $15 \mu \mathrm{M}$ (Figure 2(a), right). Thus, the increases of $\mathrm{HO}-1$ expression achieved with quercetin treatment were consistent with previous reports [24-26].

It is also known that $\mathrm{CO}$ generated by $\mathrm{HO}-1$ can activate mitochondrial biogenesis [27, 28]. Therefore, we hypothesized that the activation of mitochondrial biogenesis by quercetin also involves the activation of the $\mathrm{HO}-1 / \mathrm{CO}$ system in HepG2 cells. To investigate whether $\mathrm{HO}-1 / \mathrm{CO}$ is involved in quercetin-induced mitochondrial biogenesis, the competitive $\mathrm{HO}$ inhibitor tin-protoporphyrin-IX (SnPP) and 


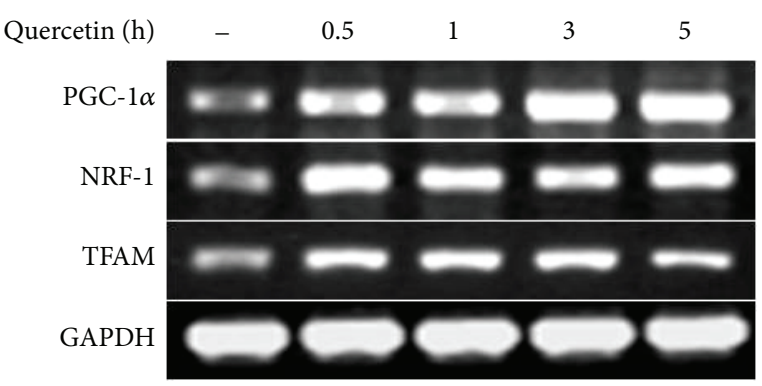

(a)

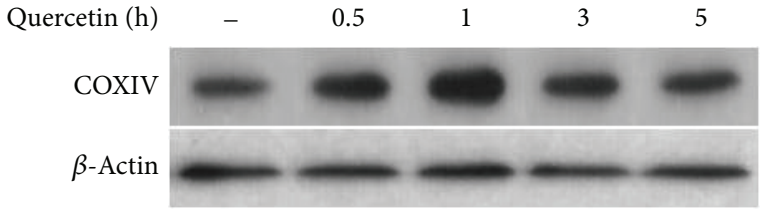

(c)

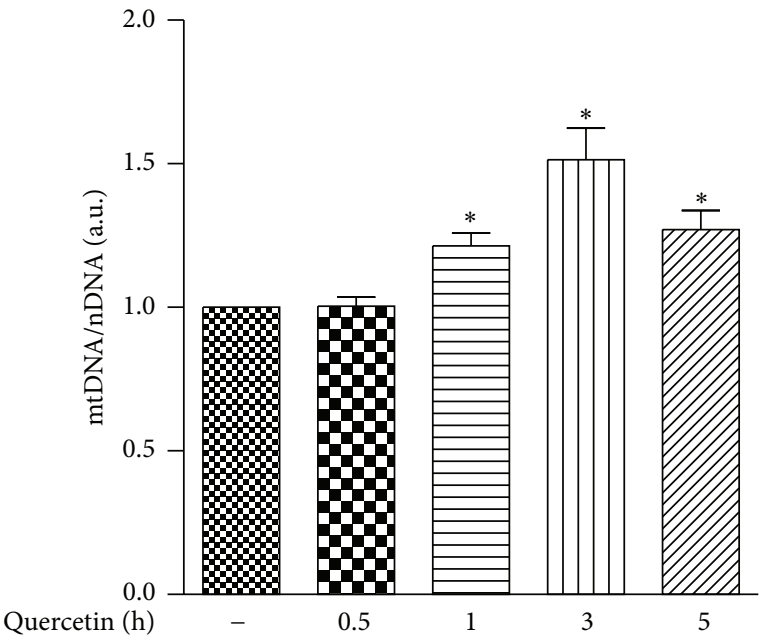

(e)

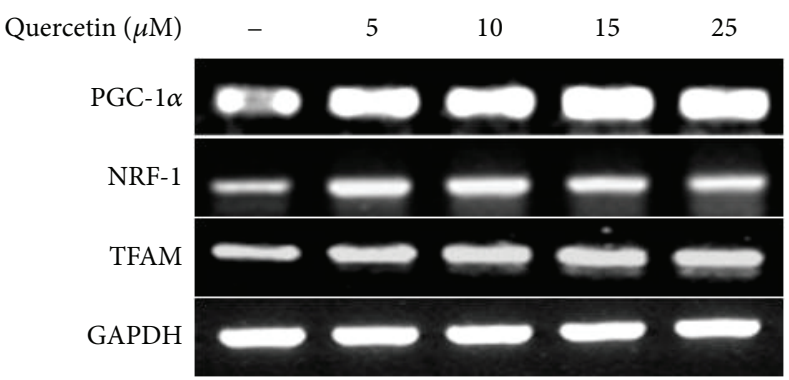

(b)

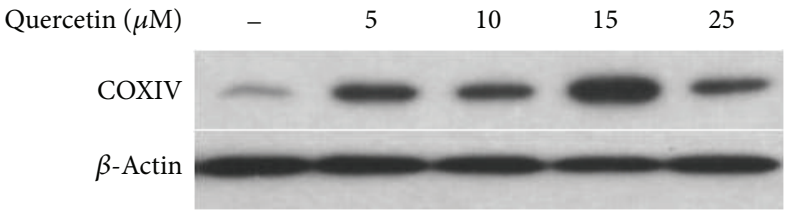

(d)

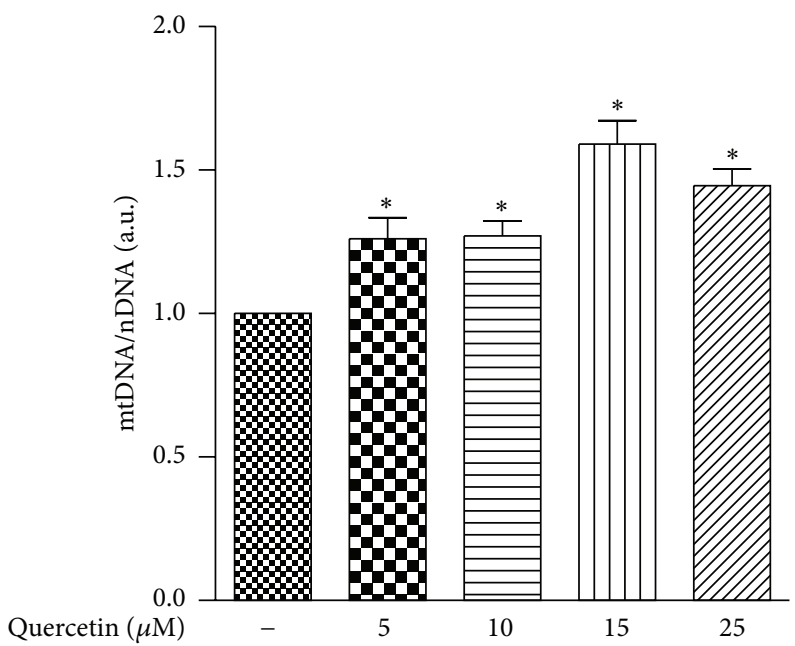

(f)

FIGURE 1: Time- and dose-dependent increases of mitochondrial biogenesis in HepG2 cells by quercetin treatment. (a-f) HepG2 cells $(4 \times$ $10^{5}$ cells/well) were exposed for indicated times $(0,0.5,1,3,5 \mathrm{~h})$ to various concentrations $(0,5,10,15,25 \mu \mathrm{M})$ of quercetin. (a, b) mRNA expressions of markers of mitochondrial biogenesis (PGC-1, NRF-1, and TFAM) were determined by reverse transcription PCR. GAPDH served as the standard. (c, d) Expression of COX IV protein was determined by Western blot analysis. $\beta$-actin served as the standard. (e, f) Expression of mitochondrial DNA (mtDNA) content was quantified by real-time PCR. Relative amount of mtDNA and nuclear DNA (nDNA) contents were compared. Results are expressed as mean \pm SE of three independent experiments, and representative data are shown. ${ }^{*} P<0.05$ compared with untreated control group. HepG2 cells $\left(4 \times 10^{5}\right.$ cells/well) were exposed to quercetin $15 \mu \mathrm{M}$, quercetin and SnPP (a), and $\mathrm{Hb}$ (b) for $3 \mathrm{~h}$ as described in panels (a) and (b). mRNA expressions of markers of mitochondrial biogenesis (PGC-1, NRF-1, and TFAM) were determined by reverse transcription (RT) PCR. Results are expressed as mean \pm SE of three independent experiments, and representative data are shown. ${ }^{*} P<0.05$ compared with untreated control group.

hemoglobin $(\mathrm{Hb})$, a CO scavenger, were employed with or without addition of quercetin, and expression levels of PGC$1 \alpha$, NRF-1, and TFAM mRNA were evaluated. As shown in Figure 2(b), treatment of HepG2 cells with SnPP and quercetin resulted in reduced levels of PGC- $1 \alpha$, NRF-1, and TFAM mRNA expression levels compared with cells treated with quercetin alone. Quercetin-induced COX IV expression was also inhibited by SnPP treatment (Figure 2(c)). Likewise, the increase in mtDNA by quercetin was suppressed by SnPP treatment (Figure 2(d)). To evaluate the involvement of CO in quercetin-induced mitochondrial biogenesis, HepG2 cells incubated with quercetin were cotreated with $\mathrm{Hb}$ (Figure 3). The $\mathrm{Hb}$ treatment inhibited the increases of PGC- $1 \alpha$, NRF-1, and TFAM mRNA expression induced by quercetin in HepG2 cells (Figure 3(a)). Hb treatment decreased the expression of COX IV protein induced by quercetin (Figure 3(b)). Furthermore, the induction of mtDNA levels by quercetin was inhibited by treatment with $\mathrm{Hb}$ (Figure 3(c)).

To examine the role of HO-1 in quercetin-induced mitochondrial biogenesis in vivo, quercetin was injected intraperitoneally in C57BL/6 mice for 7 alternate days. Mice were treated with SnPP prior to quercetin injection. In accordance 


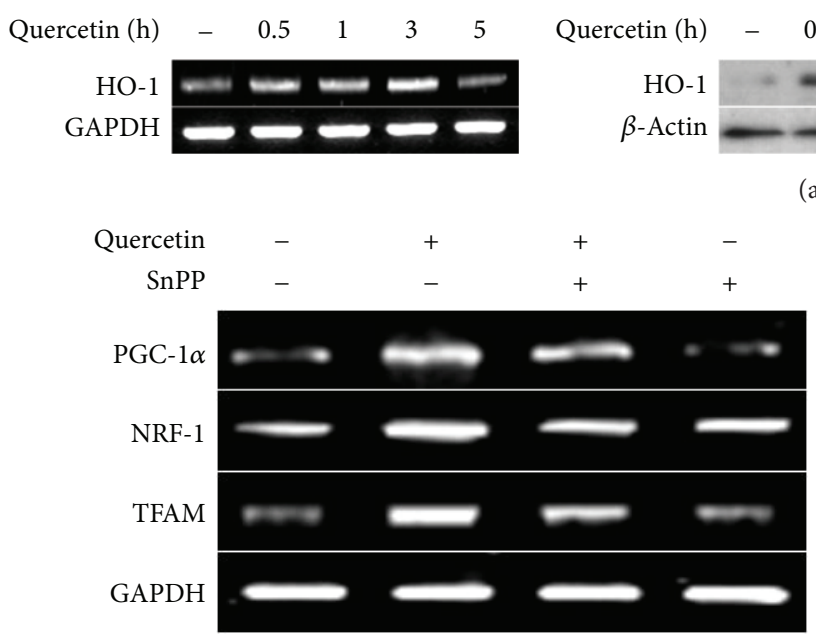

(b)

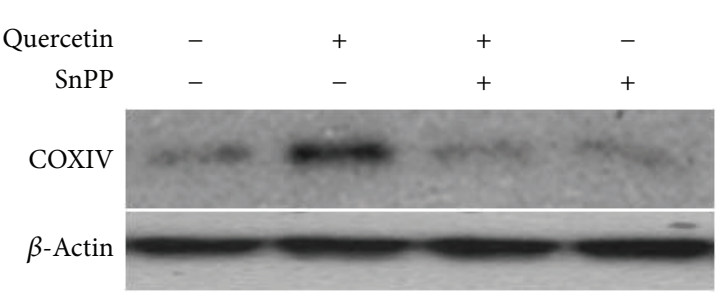

(c)

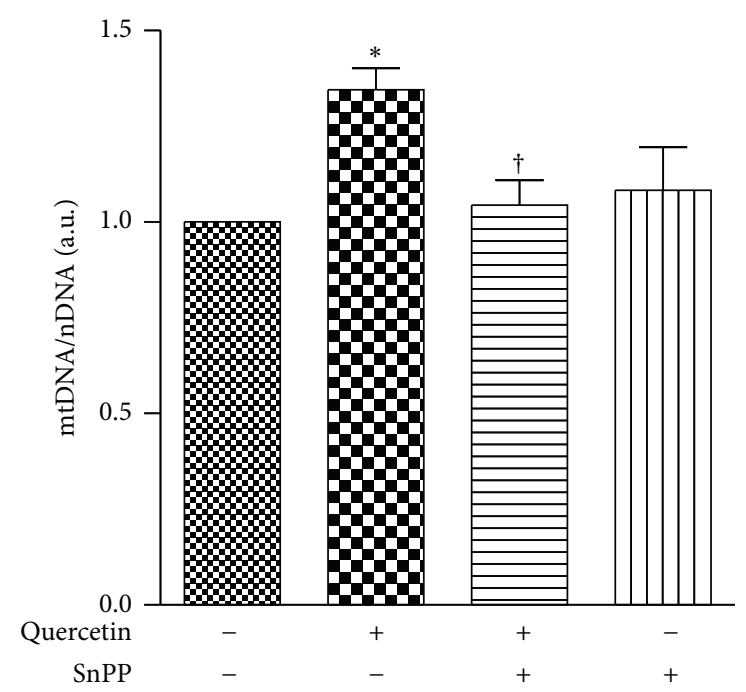

(d)

FIGURE 2: Induction of mitochondrial biogenesis by quercetin is regulated by activation of HO-1. (a) Expression levels of HO-1 mRNA and protein were determined after HepG2 cells were exposed for indicated times $(0,0.5,1,3,5 \mathrm{~h})$ and with the indicated concentrations $(0,5,10,15$, $25 \mu \mathrm{M}$ ) of quercetin. Expressions of HO-1 mRNA and protein were determined by RT-PCR and Western blotting. GAPDH and $\beta$-actin served as the standards, respectively. (b-d) HepG2 cells were exposed to $15 \mu \mathrm{M}$ of quercetin for $3 \mathrm{~h}$ with or without $20 \mu \mathrm{M}$ of SnPP. (b) Expressions of PGC-1, NRF-1, and TFAM mRNA were determined by RT-PCR. (c) Expression of COXIV protein was determined by Western blotting. (d) Expression of mtDNA content was quantified by real-time PCR. Relative amounts of mtDNA and nDNA contents were compared. Results are expressed as mean \pm SE of three independent experiments, and representative data are shown. ${ }^{*} P<0.05$ compared with untreated control group; ${ }^{\dagger} P<0.05$ compared with cells treated with quercetin alone.

with the results observed in HepG2 cells, quercetin increased the expression of PGC-1 $\alpha$, NRF-1, and TFAM mRNA, COX IV expression, and mtDNA in vivo (Figure 4 ). The cotreatment with SnPP inhibited quercetin-induced PGC- $1 \alpha$, NRF1 , and TFAM mRNA, COX IV expression, and mtDNA (Figure 4). Thus, these results suggest that HO-1/CO system is required for quercetin-induced mitochondrial biogenesis in vitro and in vivo.

3.3. Quercetin Restores LPS-Damaged Mitochondrial Integrity via HO-1/CO Induction. Finally, we examined whether quercetin could contribute to cellular protection against LPSinduced mitochondrial damage in a $\mathrm{HO}-1 / \mathrm{CO}$-dependent manner. LPS treatment increased the expression PGC$1 \alpha$, NRF-1, and TFAM mRNA in mouse liver. Moreover, quercetin treatment further increased the expression of PGC-1 $\alpha$, NRF-1, and TFAM mRNA after LPS treatment (Figure 5(a)). However, LPS treatment clearly diminished hepatic COX IV and mtDNA content (Figures 5(b) and 5(c)). In contrast, quercetin protected against the loss of COX IV and mtDNA content in LPS-treated animals. SnPP antagonized the protective effects of quercetin with respect to hepatic PGC- $1 \alpha$, NRF-1, and TFAM mRNA expression, COX 


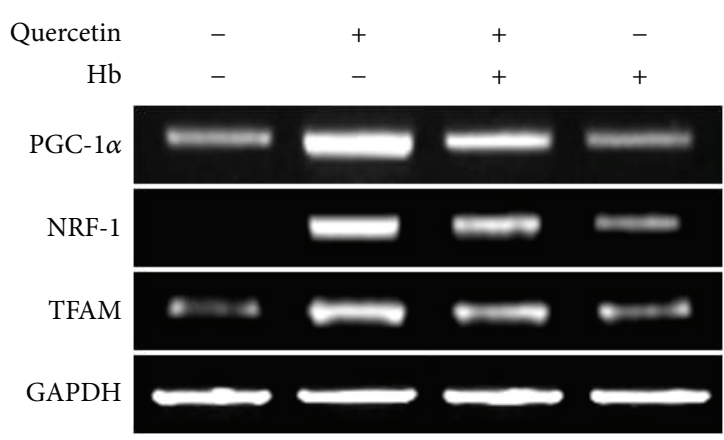

(a)

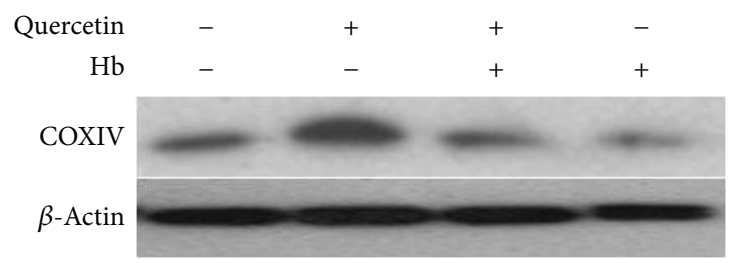

(b)

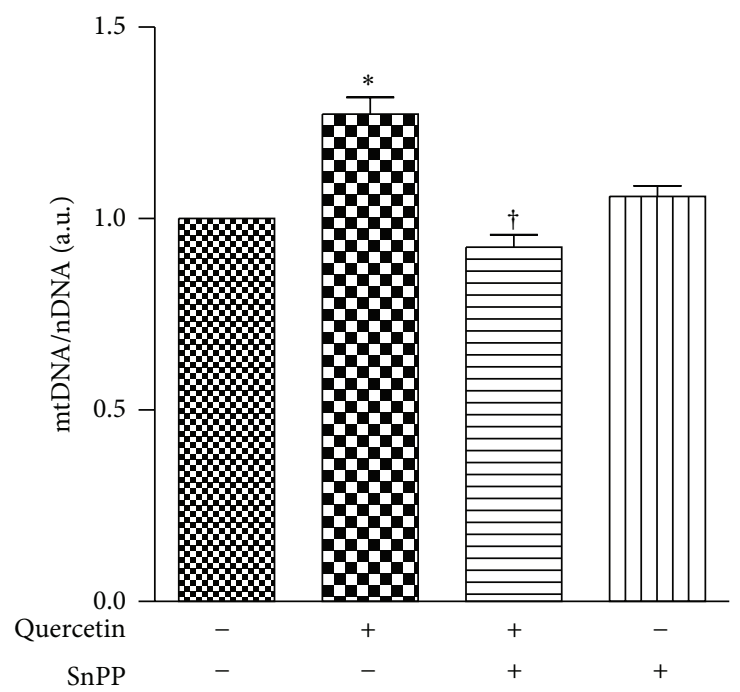

(c)

Figure 3: Quercetin induction of mitochondrial biogenesis requires CO. (a-c) HepG2 cells were exposed to $15 \mu \mathrm{M}$ of quercetin for $3 \mathrm{~h}$ with or without $20 \mu \mathrm{g} / \mathrm{mL}$ of Hb. (a) Expression of PGC-1, NRF-1, and TFAM mRNA was determined by RT-PCR. (b) Expression of COX IV protein was determined by Western blotting. $\beta$-actin served as the standard. (c) Expression of mitochondrial DNA (mtDNA) content was quantified by real-time PCR. Relative amounts of mtDNA and nDNA contents were compared. Results are expressed as mean \pm SE of three independent experiments, and representative data are shown. ${ }^{*} P<0.05$ compared with untreated control group; ${ }^{\dagger} P<0.05$ compared with cells treated with quercetin alone.

IV expression, and mtDNA content in this model (Figures 5(a), 5(b), and 5(c)). LPS caused increases in the hepatic expression of TNF $\alpha$, IL- $1 \beta$, and IL- 6 mRNA. Quercetin administration inhibited the LPS-dependent induction of TNF $\alpha$, IL-1 $\beta$, and IL- 6 . This effect of quercetin was in turn inhibited by SnPP injection (Figure 5(d)). These results suggest that quercetin restores mitochondrial integrity from LPS damage via activating the $\mathrm{HO}-1 / \mathrm{CO}$ system.

\section{Discussion}

Mitochondrial biogenesis has been the focus of extensive studies due to its beneficial effects in many health conditions related to performance, diabetes, neurodegeneration, the cardiovascular system, cancer, and infection. Death resulting from multiple organ failure (MOF) during severe sepsis and septic shock has been related to mitochondrial damage. Rescue of mice from lethal Staphylococcus aureus sepsis and protection against cardiomyocyte apoptosis have been linked to mitochondrial biogenesis induction $[27,28]$.
Quercetin is a polyphenolic compound that exerts several potent bioactivities including antiproliferative, antiinflammatory, antioxidant, and immune system effects. Recent in vitro and in vivo experiments have shown that the salutary effects of quercetin may involve activation of mitochondrial biogenesis $[11,14,15]$. Previous research has shown positive effects of quercetin on endurance and health maintenance $[4,13,29,30]$. These benefits may involve the antioxidant, anti-inflammatory, and psychostimulant effects of quercetin, as well as effects on mitochondrial biogenesis. Because abnormalities that contribute to impaired health or development of metabolic disorders are linked to mitochondrial dysfunction, the stimulation of mitochondrial biogenesis by quercetin may represent the most important bioactivity of this compound $[1,2,10,12,31]$.

Our results demonstrate that quercetin can enhance the expression of PGC- $1 \alpha$, a master regulator of the transcriptional network that regulates mitochondrial biogenesis, in HepG2 cells. PGC- $1 \alpha$ is responsible for activating the transcription of genes involved in oxidative phosphorylation 


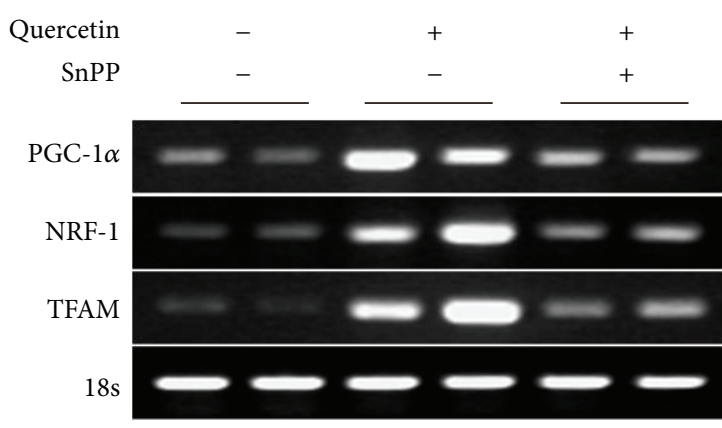

(a)

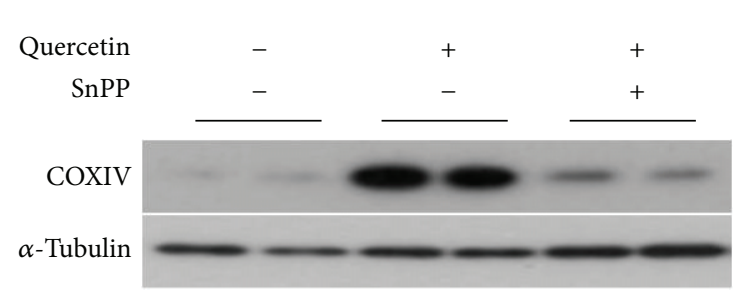

(b)

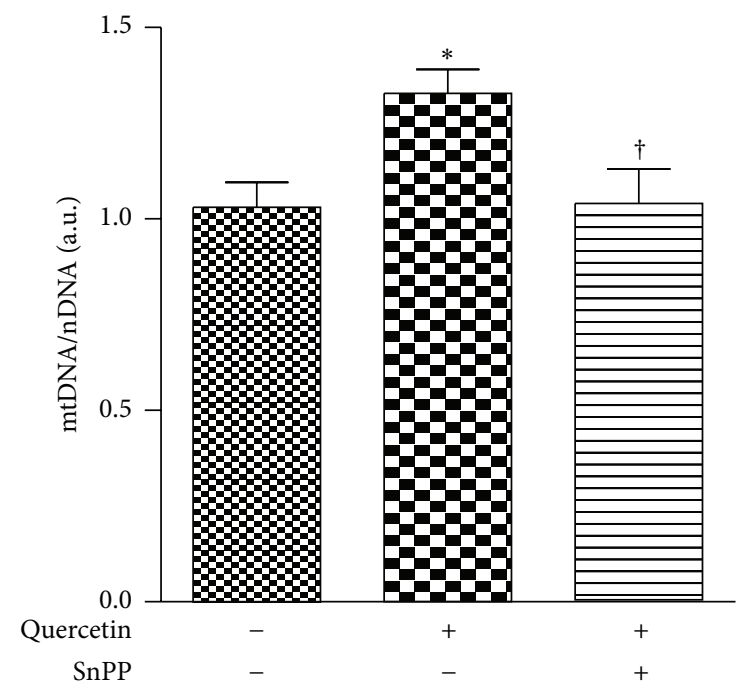

(c)

FIGURE 4: Quercetin induces mitochondrial biogenesis through the induction of HO-1/CO system in vivo. (a-c) C57BL/6 Mice were injected intraperitoneally (i.p.) with quercetin $(50 \mathrm{mg} / \mathrm{kg})$ for 7 alternate days, with or without SnPP $(50 \mu \mathrm{mol} / \mathrm{kg})$ prior to injection with the addition of quercetin. Liver tissues were excised and analyzed for mitochondrial biogenesis in mice. Experimental analyses were performed with liver tissue. (a) Expressions of PGC-1, NRF-1, and TFAM in mRNA were determined by RT-PCR. $18 \mathrm{~S}$ rRNA served as the standard. (b) Expressions of COX IV protein were determined by Western blotting. $\alpha$-tubulin served as the standard. (c) Expression of mtDNA content was quantified by real time PCR. Relative amounts of mtDNA and nDNA contents were compared. Results are expressed as mean \pm SE of three independent experiments ( $n=5$ /group), and representative data are shown. ${ }^{*} P<0.05$ compared with the uninjected control group; ${ }^{\dagger} P<0.05$ compared with LPS injected mice group.

and mtDNA replication. NRF-1 and NRF-2, which are transcription factors acting on nuclear genes coding for proteins necessary for the mitochondrial respiratory chain or for mtDNA transcription and replication, are also activated by PGC- $1 \alpha$. PGC- $1 \alpha$ and NRFs coactivate the expression of TFAM, which is important for regulation and maintenance of mtDNA copy number $[1,2,10]$. Our results also showed increased expression of PGC-1-related transcription factors associated with mitochondrial biogenesis in HepG2 cells treated with quercetin (Figure 1). Similarly, the mitochondrial respiratory chain consisting of four membrane-bound complexes (Complex I-IV) involved in ATP synthesis and transfer of electrons formed by NADH or $\mathrm{FADH}_{2}$ is an indicator of mitochondrial biogenesis [32]. Increases in cytochrome cconcentration typically occur in conjunction with similar increases in other mitochondrial enzymes of the electron transport chain and enzymes in the tricarboxylic acid cycle and $\beta$-oxidation pathway, that lead to an overall increase in mitochondrial capacity $[13,32]$. Previous reports of quercetin-induced increases in mitochondrial biogenesis are consistent with the increases in COX IV protein expression observed in our study (Figures 1-3). The critical effects of quercetin on mitochondrial biogenesis in vitro have been demonstrated at a dose of $15 \mu \mathrm{M}$ quercetin. To study the effects of quercetin in vivo, animals were treated with a $50 \mathrm{mg} / \mathrm{kg}$ dose of quercetin. According to Ruiz et al. [33], treatment of mice with up to $3000 \mathrm{mg} / \mathrm{kg}$ quercetin did not cause any toxicity. Among the doses of quercetin $(25,50$ and $100 \mathrm{mg} / \mathrm{kg})$ tested in mice, we have found that 50 and $100 \mathrm{mg} / \mathrm{kg}$ doses of quercetin have a significant effect on mitochondrial biogenesis. However, one of the limitations of the current study is that the effect of oral administration of quercetin has not been tested. Further studies would be needed to 


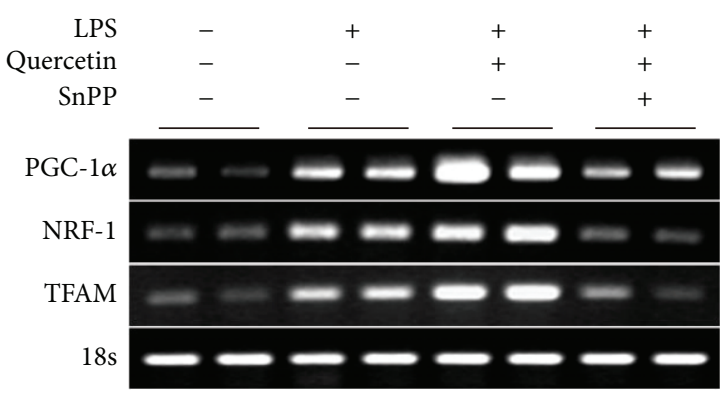

(a)

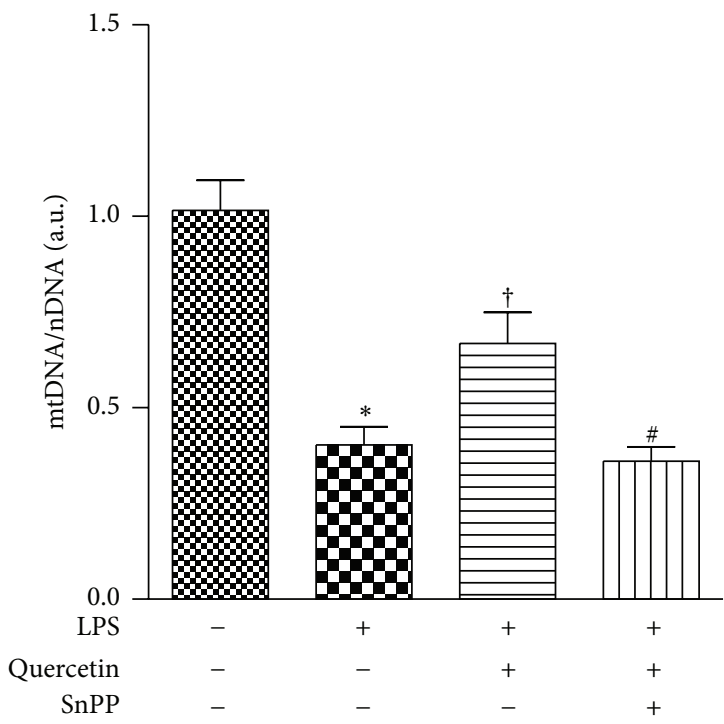

(c)

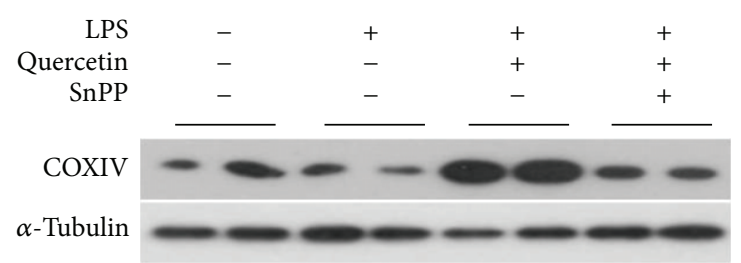

(b)

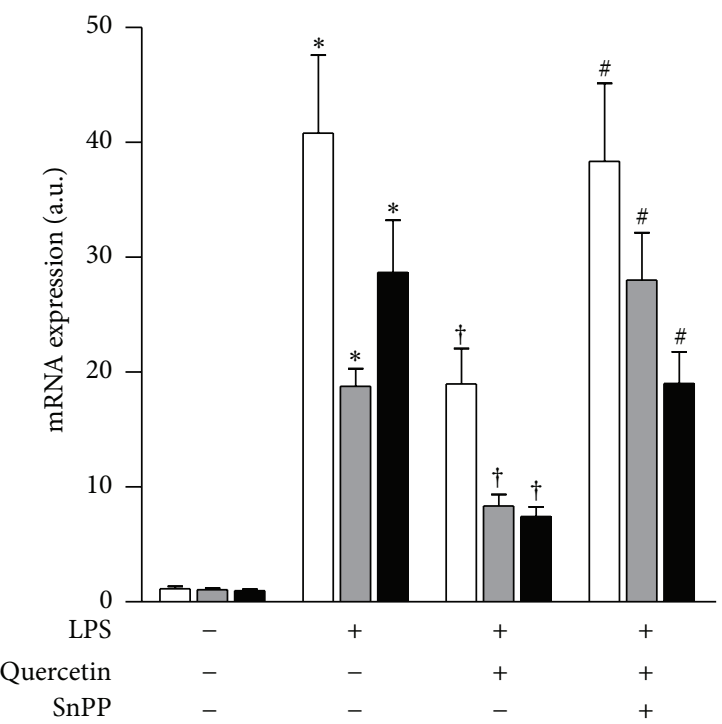

(d)

FIGURE 5: Quercetin restores mitochondrial biogenesis in LPS-treated mice in a HO-dependent fashion. (a to d) C57BL/6 mice were injected with quercetin $(50 \mathrm{mg} / \mathrm{kg})$ for 7 alternate days, with or without $S n P P(50 \mu \mathrm{mol} / \mathrm{kg})$ prior to injection with quercetin, and then challenged for 24 hours with i.p. injection of LPS (10 mg/kg). (a) Expressions of PGC-1, NRF-1, and TFAM in mRNA were determined by RT-PCR. $18 \mathrm{~S}$ rRNA served as the standard. (b) Expression of COX IV protein was determined by Western blot analysis. $\alpha$-tubulin served as the standard. (c) Expression of mtDNA content was quantified by real-time PCR. Relative amounts of mtDNA and nDNA contents were compared. (d) Expression of TNF $\alpha$, IL-1 $\beta$, and IL- 6 mRNA was quantified by real-time PCR. $18 \mathrm{~S}$ rRNA served as the standard. Results are expressed as mean \pm SE of three independent experiments $\left(n=5\right.$ /group), and representative data are shown. ${ }^{*} P<0.05$ compared with the uninjected control group; ${ }^{\dagger} P<0.05$ compared with LPS injected mice group; ${ }^{\sharp} P<0.05$ compared with mice in the LPS + quercetin group.

determine the therapeutic benefit of oral quercetin in these systems.

Recent reports have also shown that the $\mathrm{HO}-1 / \mathrm{CO}$ system can stimulate mitochondrial biogenesis which may account in part for the cytoprotective effects of this system [27, 28]. Recent research has elucidated the role of $\mathrm{HO}-1$ and $\mathrm{CO}$ in cellular defense mechanisms against oxidative damage. Quercetin has gained much attention because of its ability to confer cytoprotective effects through induction of HO-1 in various cell lines and primary hepatocytes $[9,11,13,17-$ $20,34]$. CO, an enzymatic byproduct of HO-1, can mediate the cytoprotective effects of $\mathrm{HO}-1$ activity. Our recent work has shown that endoplasmic reticulum (ER) stress caused significant decline of $\mathrm{CO}$ bioavailability that reduced mitochondrial ATP generation $[9,13,17,18,35]$. Similarly, in our current study, we have shown that the deleterious effect on mitochondria due to LPS administration was restored by quercetin. The beneficial effects of quercetin in the LPS model were in turn abrogated by SnPP. Thus, it is likely that quercetin induces mitochondrial biogenesis via the $\mathrm{HO}-1 / \mathrm{CO}$ system in HepG2 cell lines [18, 28, 35].

High glucose produces a high concentration of ROS that induces cellular dysfunction. Previous research in the human hematoma cell line, HepG2, has shown that hyperglycemia elicits detrimental changes in liver cells $[5,36]$. Increased oxidative damage caused either by an overproduction of free radicals and ROS or by an impairment of the endogenous antioxidant defense system is well studied in epithelial cells and HepG2 cells $[16,19,36]$. Prevention of lung oxidative damage in acute lung injury/acute respiratory distress syndrome by quercetin has been shown to involve increases in HO-1 production [19]. In conclusion, we demonstrated that quercetin enhances cell survival against oxidative stress through an $\mathrm{HO}-1 / \mathrm{CO}$-dependent increase in mitochondrial 
biogenesis. The antioxidant and mitochondrial biogenesis properties of quercetin may be helpful in developing therapeutic strategies to enhance cell survival during oxidative stress imposed by environmental and dietary factors.

\section{Conflict of Interests}

The authors declare that there is no conflict of interests regarding the publication of this paper.

\section{Authors' Contribution}

Nabin Rayamajhi and Seul-Ki Kim contributed equally to this paper, and thus they share first authorship.

\section{Acknowledgment}

This study was supported by the Bio \& Medical Technology Development Program of the National Research Foundation (NRF) funded by the Ministry of Science, ICT \& Future Planning (2012M3A9C3048687).

\section{References}

[1] M. B. Hock and A. Kralli, "Transcriptional control of mitochondrial biogenesis and function," Annual Review of Physiology, vol. 71, pp. 177-203, 2009.

[2] M. Lagouge, C. Argmann, Z. Gerhart-Hines et al., "Resveratrol improves mitochondrial function and protects against metabolic disease by activating SIRT1 and PGC-1 $\alpha$," Cell, vol. 127, no. 6, pp. 1109-1122, 2006.

[3] T.-K. Lin, C.-W. Liou, S.-D. Chen et al., "Mitochondrial dysfunction and biogenesis in the pathogenesis of Parkinson's disease," Chang Gung Medical Journal, vol. 32, no. 6, pp. 589-599, 2009.

[4] M. B. de Moura, L. S. dos Santos, and B. van Houten, "Mitochondrial dysfunction in neurodegenerative diseases and cancer," Environmental and Molecular Mutagenesis, vol. 51, no. 5, pp. 391-405, 2010.

[5] A. Dey and K. Swaminathan, "Hyperglycemia-induced mitochondrial alterations in liver," Life Sciences, vol. 87, no. 7-8, pp. 197-214, 2010.

[6] J. Ren, L. Pulakat, A. Whaley-Connell, and J. R. Sowers, "Mitochondrial biogenesis in the metabolic syndrome and cardiovascular disease," Journal of Molecular Medicine, vol. 88, no. 10, pp. 993-1001, 2010.

[7] J. Liu, W. Shen, B. Zhao et al., "Targeting mitochondrial biogenesis for preventing and treating insulin resistance in diabetes and obesity: hope from natural mitochondrial nutrients," Advanced Drug Delivery Reviews, vol. 61, no. 14, pp. 1343-1352, 2009.

[8] D. J. Dorta, A. A. Pigoso, F. E. Mingatto et al., "The interaction of flavonoids with mitochondria: effects on energetic processes," Chemico-Biological Interactions, vol. 152, no. 2-3, pp. 67-78, 2005.

[9] C. A. Piantadosi, M. S. Carraway, A. Babiker, and H. B. Suliman, "Heme oxygenase-1 regulates cardiac mitochondrial biogenesis via nrf2-mediated transcriptional control of nuclear respiratory factor-1," Circulation Research, vol. 103, no. 11, pp. 1232-1240, 2008.
[10] R. C. Scarpulla, "Transcriptional paradigms in mammalian mitochondrial biogenesis and function," Physiological Reviews, vol. 88, no. 2, pp. 611-638, 2008.

[11] P. Yao, A. Nussler, L. Liu et al., "Quercetin protects human hepatocytes from ethanol-derived oxidative stress by inducing heme oxygenase-1 via the MAPK/Nrf2 pathways," Journal of Hepatology, vol. 47, no. 2, pp. 253-261, 2007.

[12] M. B. Hock and A. Kralli, "Transcriptional control of mitochondrial biogenesis and function," Annual Review of Physiology, vol. 71, pp. 177-203, 2009.

[13] J. M. Davis, E. A. Murphy, M. D. Carmichael, and B. Davis, "Quercetin increases brain and muscle mitochondrial biogenesis and exercise tolerance," American Journal of Physiology: Regulatory Integrative and Comparative Physiology, vol. 296, no. 4, pp. R1071-R1077, 2009.

[14] M. Gross, M. Pfeiffer, M. Martini, D. Campbell, J. Slavin, and J. Potter, "The quantitation of metabolites of quercetin flavonols in human urine," Cancer Epidemiology Biomarkers and Prevention, vol. 5, no. 9, pp. 711-720, 1996.

[15] M. Harwood, B. Danielewska-Nikiel, J. F. Borzelleca, G. W. Flamm, G. M. Williams, and T. C. Lines, "A critical review of the data related to the safety of quercetin and lack of evidence of in vivo toxicity, including lack of genotoxic/carcinogenic properties," Food and Chemical Toxicology, vol. 45, no. 11, pp. 2179-2205, 2007.

[16] H. Kaneto, N. Katakami, D. Kawamori et al., "Involvement of oxidative stress in the pathogenesis of diabetes," Antioxidants and Redox Signaling, vol. 9, no. 3, pp. 355-366, 2007.

[17] F. A. Wagener, H.-D. Volk, D. Willis et al., "Different faces of the heme-heme oxygenase system in inflammation," Pharmacological Reviews, vol. 55, no. 3, pp. 551-571, 2003.

[18] S. W. Ryter, J. Alam, and A. M. Choi, "Heme oxygenase-1/carbon monoxide: from basic science to therapeutic applications," Physiological Reviews, vol. 86, no. 2, pp. 583-650, 2006.

[19] Y. Hayashi, M. Matsushima, T. Nakamura et al., "Quercetin protects against pulmonary oxidant stress via heme oxygenase1 induction in lung epithelial cells," Biochemical and Biophysical Research Communications, vol. 417, no. 1, pp. 169-174, 2012.

[20] B.-M. Choi, H.-O. Pae, Y.-M. Kim, and H.-T. Chung, "Nitric oxide-mediated cytoprotection of hepatocytes from glucose deprivation-induced cytotoxicity: involvement of heme oxygenase-1," Hepatology, vol. 37, no. 4, pp. 810-823, 2003.

[21] J.-M. Chow, S.-C. Shen, S. K. Huan, H.-Y. Lin, and Y.-C. Chen, "Quercetin, but not rutin and quercitrin, prevention of $\mathrm{H}_{2} \mathrm{O}_{2}$ induced apoptosis via anti-oxidant activity and heme oxygenase 1 gene expression in macrophages," Biochemical Pharmacology, vol. 69, no. 12, pp. 1839-1851, 2005.

[22] H. K. Park, S. J. Kim, D. Y. Kwon, J. H. Park, and Y. C. Kim, "Protective effect of quercetin against paraquat-induced lung injury in rats," Life Sciences, vol. 87, no. 5-6, pp. 181-186, 2010.

[23] H.-Y. Lin, S.-H. Juan, S.-C. Shen, F.-L. Hsu, and Y.-C. Chen, "Inhibition of lipopolysaccharide-induced nitric oxide production by flavonoids in RAW264.7 macrophages involves heme oxygenase-1," Biochemical Pharmacology, vol. 66, no. 9, pp. 18211832, 2003.

[24] P. Yao, L. Hao, N. Nussler et al., "The protective role of $\mathrm{HO}-1$ and its generated products ( $\mathrm{CO}$, bilirubin, and $\mathrm{Fe}$ ) in ethanol-induced human hepatocyte damage," American Journal of Physiology: Gastrointestinal and Liver Physiology, vol. 296, no. 6, pp. G1318-G1323, 2009. 
[25] A. K. Nussler, L. Hao, D. Knobeloch et al., "Protective role of HO-1 for alcohol-dependent liver damage," Digestive Diseases and Sciences, vol. 28, no. 6, pp. 792-798, 2010.

[26] S. Liu, W. Hou, P. Yao et al., "Heme oxygenase-1 mediates the protective role of quercetin against ethanol-induced rat hepatocytes oxidative damage," Toxicology in Vitro, vol. 26, no. 1, pp. 74-80, 2012.

[27] N. C. MacGarvey, H. B. Suliman, R. R. Bartz et al., "Activation of mitochondrial biogenesis by heme oxygenase-1-mediated NF-E2-related factor-2 induction rescues mice from lethal Staphylococcus aureus sepsis," American Journal of Respiratory and Critical Care Medicine, vol. 185, no. 8, pp. 851-861, 2012.

[28] H. B. Suliman, M. S. Carraway, A. S. Ali, C. M. Reynolds, K. E. Welty-Wolf, and C. A. Piantadosi, "The CO/HO system reverses inhibition of mitochondrial biogenesis and prevents murine doxorubicin cardiomyopathy," Journal of Clinical Investigation, vol. 117, no. 12, pp. 3730-3741, 2007.

[29] H. S.-H. MacRae and K. M. Mefferd, "Dietary antioxidant supplementation combined with quercetin improves cycling time trial performance," International Journal of Sport Nutrition and Exercise Metabolism, vol. 16, no. 4, pp. 405-419, 2006.

[30] S. Egert, S. Wolffram, A. Bosy-Westphal et al., "Daily quercetin supplementation dose-dependently increases plasma quercetin concentrations in healthy humans," Journal of Nutrition, vol. 138, no. 9, pp. 1615-1621, 2008.

[31] J. M. Davis, E. A. Murphy, and M. D. Carmichael, "Effects of the dietary flavonoid quercetin upon performance and health," Current Sports Medicine Reports, vol. 8, no. 4, pp. 206-213, 2009.

[32] A. Lemarie and S. Grimm, "Mitochondrial respiratory chain complexes: apoptosis sensors mutated in cancer," Oncogene, vol. 30, no. 38, pp. 3985-4003, 2011.

[33] M. J. Ruiz, M. Fernández, Y. Picó et al., "Dietary administration of high doses of pterostilbene and quercetin to mice is not toxic," Journal of Agricultural and Food Chemistry, vol. 57, no. 8, pp. 3180-3186, 2009.

[34] M. D. Maines, "The heme oxygenase system: a regulator of second messenger gases," Annual Review of Pharmacology and Toxicology, vol. 37, pp. 517-554, 1997.

[35] M. Zheng, S. K. Kim, Y. Joe et al., "Sensing endoplasmic reticulum stress by protein kinase RNA-like endoplasmic reticulum kinase promotes adaptive mitochondrial DNA biogenesis and cell survival via heme oxygenase 1/carbon monoxide activity," Federation of American Societies for Experimental Biology, vol. 26, no. 6, pp. 2558-2568, 2012.

[36] K. Chandrasekaran, K. Swaminathan, S. Chatterjee, and A. Dey, "Apoptosis in HepG2 cells exposed to high glucose," Toxicology in Vitro, vol. 24, no. 2, pp. 387-396, 2010. 


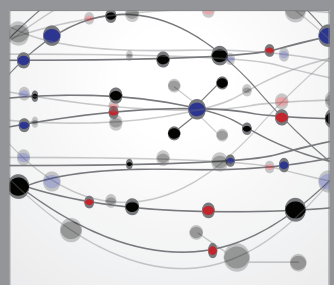

The Scientific World Journal
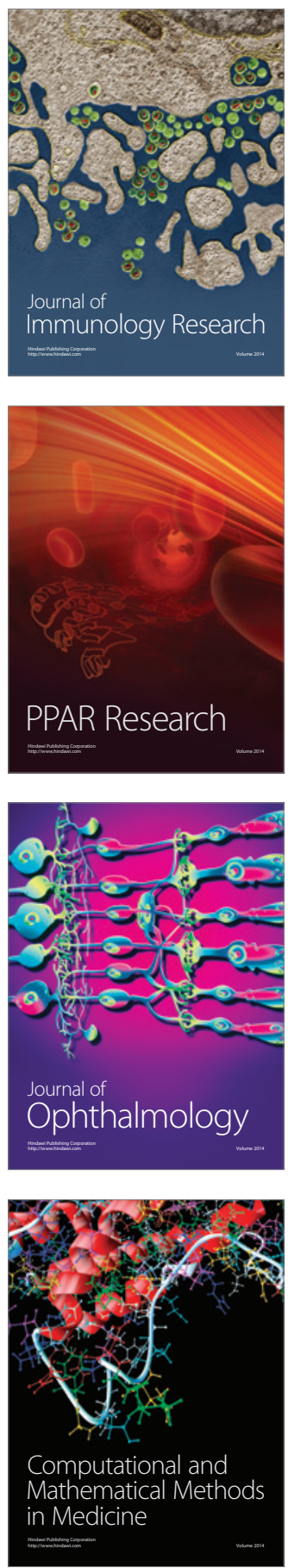

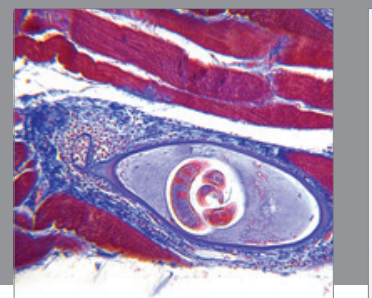

Gastroenterology

Research and Practice
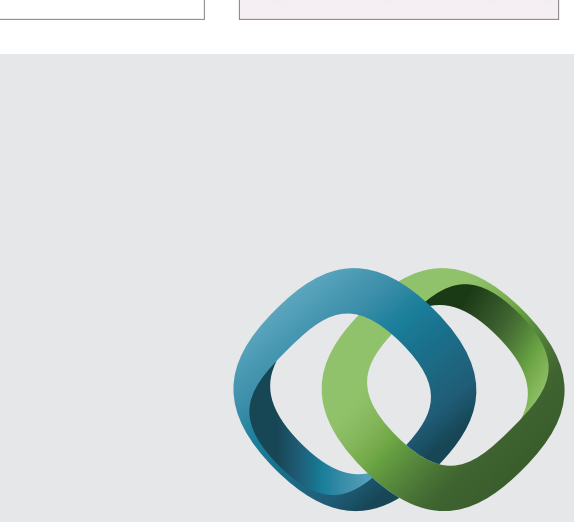

\section{Hindawi}

Submit your manuscripts at

http://www.hindawi.com
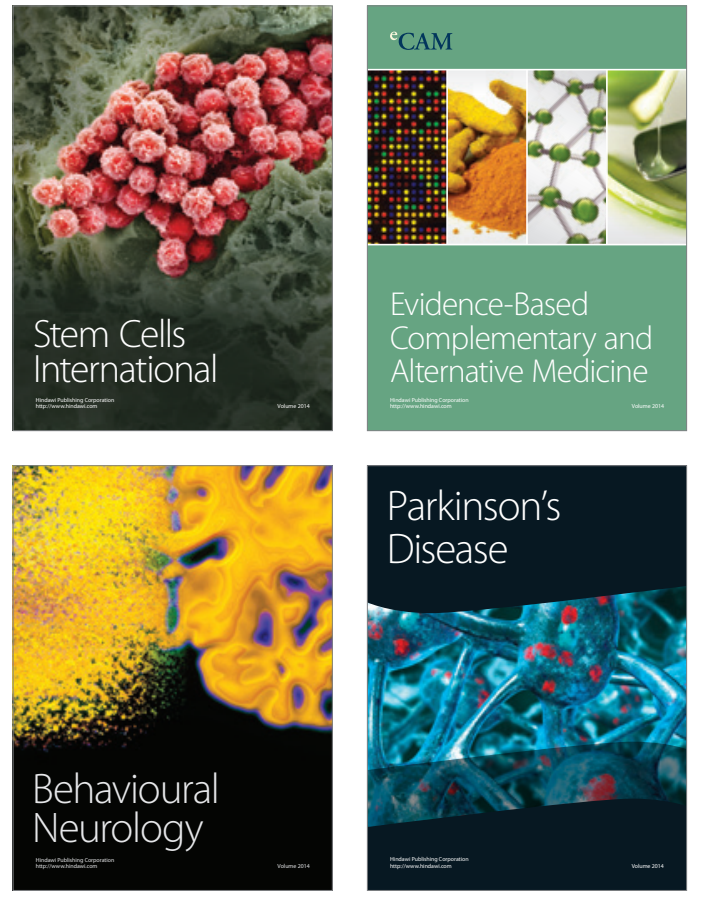
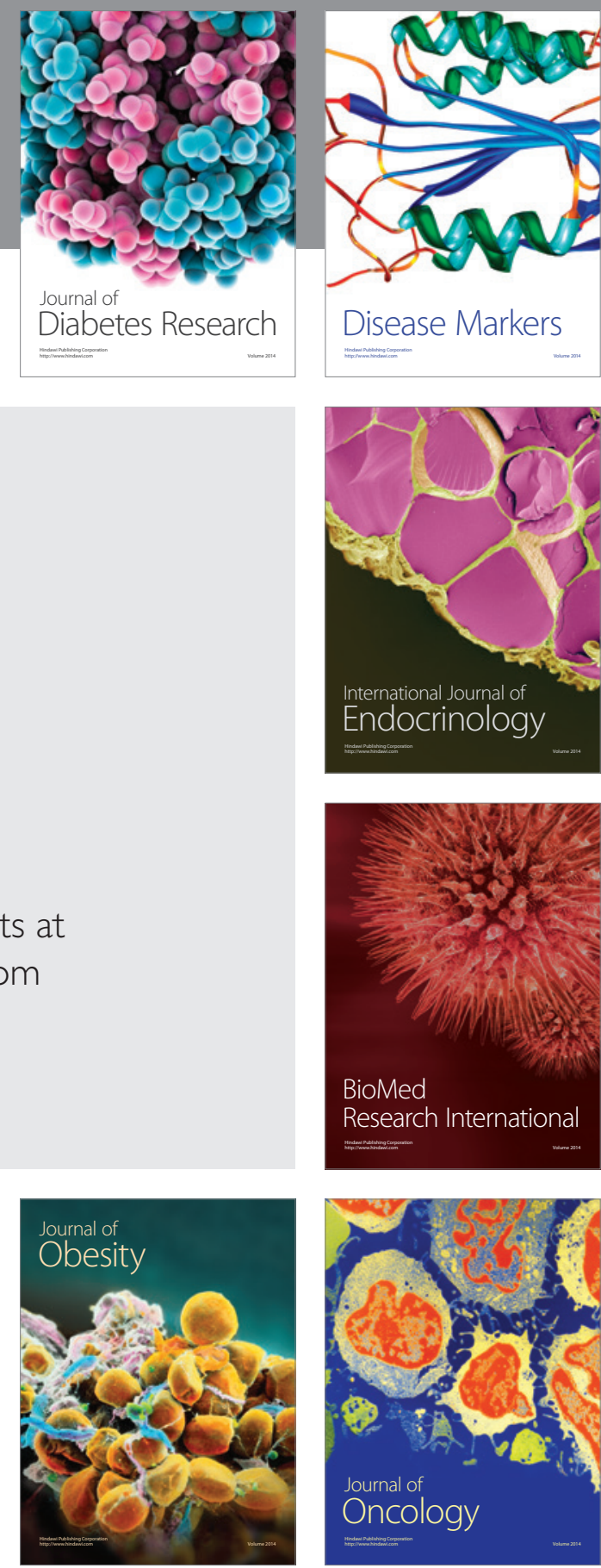

Disease Markers
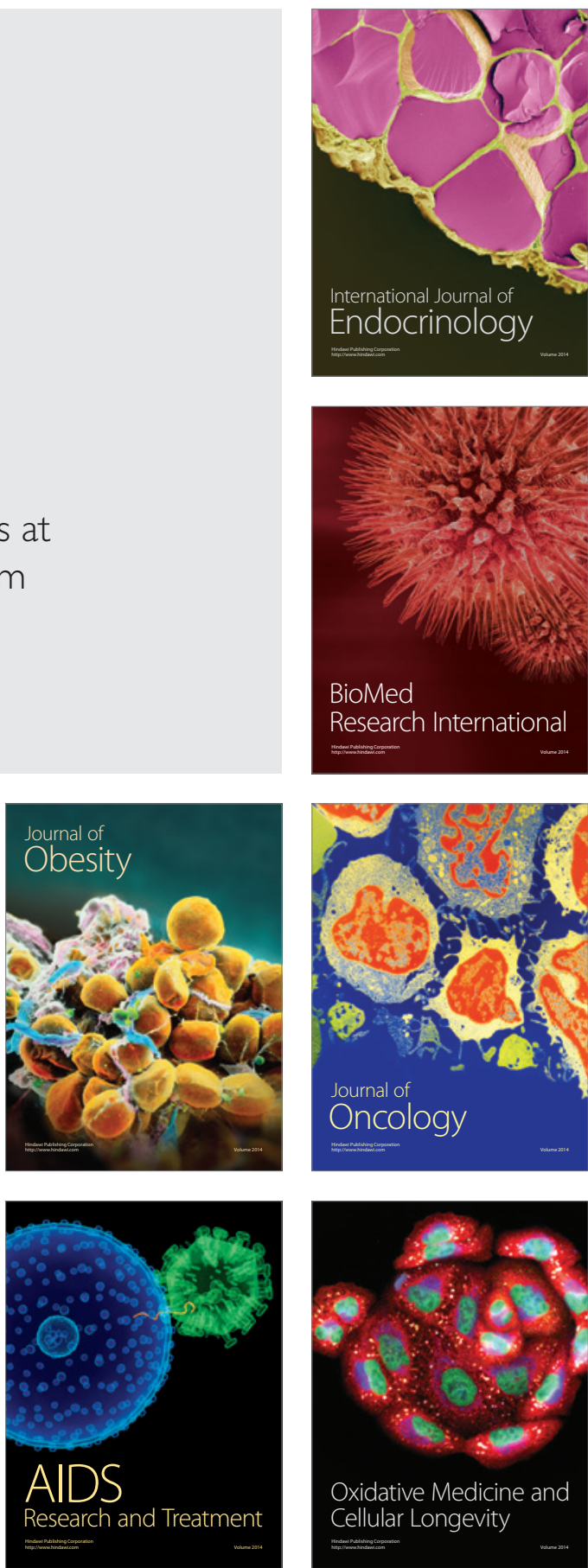